\title{
ELECTRICAL CONDUCTIVITY OF THIN WIRES
}

\author{
R. DIMMICH and F. WARKUSZ
}

Institute of Physics, Technical University of Wroclaw Wybrzeze Wyspianskiege 27, 50-370 Wroclaw, Poland

(Received December 13, 1982; in final form November 14, 1984)

An expression for the electrical conductivity of thin metal wires of a circular cross-section has been derived taking account of conduction-electron scattering at grain boundaries and the wire surface. An angular-dependent specularity parameter has been introduced and possible fluctuations of the wire diameter along its length have been taken into account. Boltzmann transport equations have been solved for the boundary conditions proposed by Dingle.

\section{INTRODUCTION}

The electrical conductivity of thin metal wires has been investigated many times in the past (see the review by Larson $^{1}$ ). The experimental data were interpreted mainly on the basis of Dingle's theory ${ }^{2}$ for wires of a circular cross-section. In this theory, electron-carrier scattering at the wire surface was taken into account by introducing a $\mathrm{p}$ parameter which represents the fraction of conduction electrons specularly reflected at the external surface. Recently, Sambles et al. ${ }^{3}$ developed Dingle's theory based, when describing the scattering at the wire surfaces, on suggestions given by Ziman ${ }^{4}$ and on Soffer's later model. ${ }^{5}$

They introduced the angular-dependent specularity parameter, $p(\theta)$, dependent upon the angle of incidence, $(\theta)$, of electrons to the surface normal. New theoretical approaches of Sambles et al. to the earlier experimental data for conductivity of thin wires led to qualitatively novel conclusions. ${ }^{3}$ However, both the theories do not take account of the polycrystalline metal structure, and therefore of electronzarrier scattering at the grain boundaries. As was shown by Mayadas and Shatzkes ${ }^{6}$ this factor essentially modifies the electric conductivity of the thin films. Hence an analogous influence may be expected in the case of thin wires. The latest experimental studies of ultra-thin drawn wires (Sacharoff et al. ${ }^{7}$ ) have shown that fluctuations of the wire diameter along its length can reach $40 \%$. It is certain (see results of Namba, ${ }^{8}$ Elsom and Sambles ${ }^{9}$ for thin films) that this factor also modifies electric conductivity of thin wires.

In this work Sambles' model ${ }^{3}$ has been developed taking account of both the above problems.

\section{THEORY}

Our samiple of the wire is placed, let us say, along the z-direction. Then the distribution function, $f$, of conduction electrons depends upon $x-$ and $y$-coordinates and upon electron-velocity vector, $\bar{v}$, i.e.

$$
f=f_{0}+f_{1}(\bar{v}, x, y)
$$

and for low electric fields, $E$, the function satisfies the following Boltzmann equation:

$$
v_{\mathrm{x}} \frac{\partial \mathrm{f}_{1}}{\partial \mathrm{x}}+v_{\mathrm{y}} \frac{\partial \mathrm{f}_{1}}{\partial \mathrm{y}}+\frac{\mathrm{f}_{1}}{\tau}=\frac{\mathrm{eE}}{\mathrm{m}_{\mathrm{e}}} \frac{\partial \mathrm{f}_{0}}{\partial v_{\mathrm{z}}}
$$


where $\mathrm{f}_{0}$ denotes the Fermi-Dirac distribution equilibrium function, $\mathrm{e}$ is the absolute value of electron charge, $\mathrm{m}_{\mathrm{e}}$ - electron mass, $\tau$ - electron relaxation time.

In a cylindrical coordinate system Eq. (1) has the form:

$v_{\mathrm{r}} \frac{\partial \mathrm{f}_{1}}{\partial \mathrm{r}}+\frac{v_{\theta}^{2}}{\mathrm{r}} \frac{\partial \mathrm{f}_{1}}{\partial v_{\mathrm{r}}}-\frac{v_{\theta} v_{\mathrm{r}}}{\mathrm{r}} \frac{\partial \mathrm{f}_{1}}{\partial v_{\theta}}+\frac{\mathrm{f}_{1}}{\tau}=\frac{\mathrm{eE}}{\mathrm{m}_{\mathrm{e}}} \frac{\partial \mathrm{f}_{0}}{\partial v_{\mathrm{z}}}$

Dingle's boundary conditions ${ }^{2}$ together with the angular dependence of the specularity parameter given by Soffers, ${ }^{3}$ i.e.

$p(\theta)=\exp \left[-(4 \pi H)^{2} \sin ^{2} \theta \sin ^{2} \varphi\right]$

are the following:-

$f_{1}\left(-v_{r}, v_{\theta}, v_{a}, r=a\right)=p f_{1}\left(v_{r}, v_{\theta}, v_{z}, r=a\right)$

where $H=h / \lambda_{.} . h$ is a root-mean-square surface roughness, $\lambda_{e}$ is the electron wavelength, and a denotes the wire radius,

$v_{\mathrm{r}_{1}}, v_{\phi}$ and $v_{\mathrm{z}}$ are connected by:-

$\cos ^{2} \theta=v_{\mathrm{r}}^{2} /\left(v_{\mathrm{r}}^{2}+v_{\theta}^{2}+v_{\mathrm{z}}^{2}\right)$

Considering the boundary conditions (Eq. (4)) distribution function, $\mathrm{f}_{1}$, has been calculated by solving Eq. (1) and the electric conductivity, $\sigma$, of the wire has been also calculated ${ }^{3}$ :

$$
\begin{aligned}
\frac{\sigma}{\sigma_{\infty}} & =1-\frac{12}{\pi \mathrm{k}} \int_{0}^{\pi / 2} \cos ^{2} \theta \sin ^{2} \theta \mathrm{d} \theta \int_{0}^{\pi / 2} \sin \varphi \mathrm{d} \varphi \\
& \times \frac{(1-\mathrm{p}(\theta))[1-\exp (-\mathrm{k} \sin \varphi / \sin \theta)]}{[1-\mathrm{p}(\theta) \exp (-\mathrm{k} \sin \varphi / \sin \theta)]}
\end{aligned}
$$

where $\mathrm{k}=2 \mathrm{a} / \lambda_{\infty}, \lambda_{\infty}$ is an electron mean free path for bulk metal, and $\sigma_{\infty}$ is the electric conductivity of bulk metal, and the specularity parameter, $p(\theta)$, for reflection at the outer surface varies from 0 to 1 . When $h / \lambda_{e}$ tends to infinity, $p(\theta)$ approaches zero and then we have total diffusive reflection which consists in the limit of a drift velocity at the film surface.

Neither electron scattering at grain boundaries nor the influence of thin-wire diameter fluctuations along the wire length have been considered previously. Our work takes account of these two problems.

\subsection{Grain Boundary}

If a wire is composed of polycrystalline metal, it possesses in principle grain structure, and grain boundaries have an essential influence on electric conductivity. This problem was partially solved by Mayadas-Shatzkes ${ }^{6}$ for thin metallic films. Grain boundaries perpendicular to the electric-current flow are considered. Taking account of electron scattering at grain boundaries, relaxation time takes the following form:-

$\frac{1}{\tau_{\mathrm{D}}}=\frac{1}{\tau} \mathcal{H C}(\alpha, \theta)$ 
where $\mathcal{H}(\alpha, \theta)=1+\frac{\alpha}{\cos \theta}$

$$
\alpha=\frac{\lambda_{\infty}}{\mathrm{D}} \frac{\mathrm{R}^{*}}{1-\mathrm{R}^{*}}
$$

$\mathrm{D}$ is an average distance between grain boundaries (in experiments it is frequently taken as the grain size), $R^{*}$ is the electron-reflection parameter at grain boundaries, $0 \leqslant R^{*} \leqslant 1$.

$\mathrm{R}^{*}$ is defined such that $\mathrm{R}^{*}=0$ for entire transmission of electrons through grain boundaries, and $\mathrm{R}^{*}=1$ when total electron reflection at a grain boundary occurs.

It should be noted that the reflection or scattering processes at grain boundaries and outer surfaces of the wire occur independently (see e.g. Eqs. (6) (7)). $\tau_{\mathrm{D}}$ is substituted for $\tau$ into Eq. (1) and $f_{1}$ is calculated. Dingle's boundary conditions are taken into account (Eq. (4)). Under these conditions electric conductivity has the following form:-

$$
\begin{aligned}
\frac{\sigma}{\sigma_{\infty}}= & \mathrm{f}(\alpha)-\frac{12}{\pi \mathrm{k}} \int_{0}^{1} \frac{\mathrm{t}^{2} \sqrt{ } 1-\mathrm{t}^{2}}{\mathcal{H}^{2}(\alpha, \mathrm{t})} \mathrm{dt} \int_{0}^{\pi / 2} \sin \varphi \mathrm{d} \varphi \\
& \times \frac{(1-\mathrm{p}(\theta))\left[1-\exp \left(-\frac{\mathrm{k} \mathcal{C}(\alpha, \mathrm{t})}{\sqrt{ } 1-\mathrm{t}^{2}} \sin \varphi\right)\right]}{1-\mathrm{p}(\theta) \exp \left(-\frac{\mathrm{k} \mathcal{H}(\alpha, \mathrm{t})}{\sqrt{ } 1-\mathrm{t}^{2}} \sin \varphi\right)}=\mathrm{G}(\mathrm{k}, \mathrm{H}, \alpha)
\end{aligned}
$$

where $f(\alpha)=3\left[\frac{1}{3}-\frac{1}{2} \alpha+\alpha^{2}-\alpha^{3} \ln \left(1+\frac{1}{\alpha}\right)\right]$

$\alpha$ is given by Eq. (8). $t=\cos \theta$, and $\mathrm{p}(\theta)$ is given by Eq. (3).

For the total mirror-like reflection of electrons at outer surfaces, i.e. $p(\theta)=1$, Eq. (9) becomes Eq. (10). However, this process is highly unlikely.

If we neglect electron scattering at grain boundaries, then Eq. (10) equals unity $(\mathrm{f}(\alpha)=1)$ and $\mathcal{H}(\alpha, \mathrm{t})=1$ and Eq. (9) becomes Eq. (5).

\subsection{Wire-diameter Fluctuations}

It is unquestionable that an essential influence on the conductivity value is exerted by diameter fluctuations of a very thin wire along its length. ${ }^{7,8}$ Using the approach given by $\mathrm{Namba}^{8}$ for thin films we denote the wire diameter by $\mathrm{d}=2 \mathrm{a}$, so that $\mathrm{k}=2 \mathrm{a} / \lambda_{\infty}=\mathrm{d} / \lambda_{\infty}$. Let us denote also the diameter-fluctuation amplitude by $\mathrm{g}$ and the ratio of $g$ to the electron mean free path in the bulk by $B$, i.e. $B=g / \lambda_{\infty}$. Let us assume that the wire diameter to change as follows:-

$\mathrm{d}=\mathrm{d}_{\mathrm{average}}+\mathrm{gsin} \frac{2 \pi}{1} \mathrm{z}$

where $d_{\text {average }}$ is the average value of $d, l$ is the wire length. Assumption (11) is a certain simplification and an idealized way of the diameter fluctuating. In Eq. (11) we have considered only the first term of the Fourier series:- 
$\mathrm{d}=\mathrm{d}_{\mathrm{av}}+\mathrm{g} \sin \frac{2 \pi}{1} \mathrm{z}+\sum_{\mathrm{n}=2}^{\infty} \mathrm{g}_{\mathrm{n} \sin }\left(\frac{2 \pi \eta}{1} \mathrm{z}+\varphi_{\mathrm{n}}\right)$

The wire resistance, $R$, may be expressed with aid of the integral:-

$R=\int_{0}^{1} \frac{\rho(d)}{\frac{\pi}{4} d^{2}} d z$

where the diameter $\mathrm{d}$ is a function of $\mathrm{z}$ (Eq. (11)). Total resistance of the wire may be connected with the average resistivity, $\rho\left(\mathrm{d}_{\mathrm{av}}\right)$ by means of:-

$\mathrm{R}=\frac{\rho\left(\mathrm{d}_{\mathrm{av}}\right)}{\frac{\pi}{4} \mathrm{~d}_{\mathrm{av}}^{2}} 1$

hence

$\rho\left(\mathrm{d}_{\mathrm{av}}\right)=\frac{\pi \mathrm{R}}{41} \mathrm{~d}_{\mathrm{av}}^{2}$

Substituting the expression for R from Eq. (13) into Eq. (15) we obtain:-

$\rho\left(\mathrm{d}_{\mathrm{av}}\right)=\frac{\mathrm{d}_{\mathrm{av}}^{2}}{1} \int_{0}^{1} \frac{\rho(\mathrm{d})}{\mathrm{d}^{2}} \mathrm{dz}$

or, dividing by $\lambda_{\infty}$ and $\rho_{\infty}$ :-

$\frac{\rho\left(\mathrm{d}_{\mathrm{av}}\right)}{\rho_{\infty}}=\frac{\mathrm{d}_{\mathrm{av}}^{2} / \lambda_{\infty}^{2}}{\rho_{\infty} 1} \int_{0}^{1} \frac{\rho\left(\mathrm{d}_{\mathrm{av}} / \lambda_{\infty}+\left(\mathrm{g} / \lambda_{\infty}\right) \sin \frac{2 \pi}{1} \mathrm{z}\right)}{\left(\mathrm{d}_{\mathrm{av}} / \lambda_{\infty}+\left(\mathrm{g} / \lambda_{\infty}\right) \sin \frac{2 \pi}{1} \mathrm{z}\right)^{2}} \mathrm{dz}$

In the further calculations we use the following notation

$\frac{\rho\left(\mathrm{d}_{\mathrm{av}}\right)}{\rho_{\infty}}=\frac{\rho}{\rho_{\infty}}, \mathrm{k}=\frac{\mathrm{d}_{\mathrm{av}}}{\lambda_{\infty}}, \phi=\frac{2 \pi}{1} \mathrm{z}, \mathrm{B}=\frac{\mathrm{g}}{\lambda_{\infty}}, \mathrm{T}=\frac{\mathrm{D}}{\lambda_{\infty}}$

Then Eq. (17) has the form

$\frac{\rho}{\rho_{\infty}}=\frac{\mathrm{k}^{2}}{\rho_{\infty} 2 \pi} \int_{0}^{2 \pi} \frac{\rho(\mathrm{k}+\mathrm{B} \sin \phi)}{(\mathrm{k}+\mathrm{B} \sin \phi)^{2}} \mathrm{~d} \phi$

The Ratio

$\frac{\rho_{\infty}}{\rho(\mathrm{k}+\mathrm{B} \sin \phi)}=\frac{\sigma(\mathrm{k}+\mathrm{B} \sin \phi)}{\sigma_{\infty}}$ 
is obtained by substituting values of $\mathrm{k}+\mathrm{B} \sin \phi$ for $\mathrm{k}$ in Eq. (9), which means

$\mathrm{G}(\mathrm{k}, \mathrm{H}, \alpha) \Rightarrow \mathrm{G}(\mathrm{k}+\mathrm{B} \sin \phi, \mathrm{H}, \alpha)$

Substituting $\mathrm{G}(\mathrm{k}+\mathrm{B} \sin \phi, \mathrm{H}, \alpha)$ into Eq. (18) we obtain

$\frac{\rho}{\rho_{\infty}}=\frac{\mathrm{k}^{2}}{2 \pi} \int_{0}^{2 \pi}\left[(\mathrm{k}+\mathrm{B} \sin \phi)^{2} \mathrm{G}(\mathrm{k}+\mathrm{B} \sin \phi, \mathrm{H}, \alpha)\right]^{-1} \mathrm{~d} \phi$

This is the most general equation for calculating the thin-wire resistivity, if electron scattering occurs at the outer surfaces of a rough modulated structure and at the grain boundaries.

\section{DISCUSSION AND CONCLUSIONS}

The transport theory for thin wires presented in Section 2 is based on the Boltzmann equation (Eq. (1)) with the appropriately chosen boundary conditions (Eq. (4)). When calculating electric conductivity of thin wires we have considered,

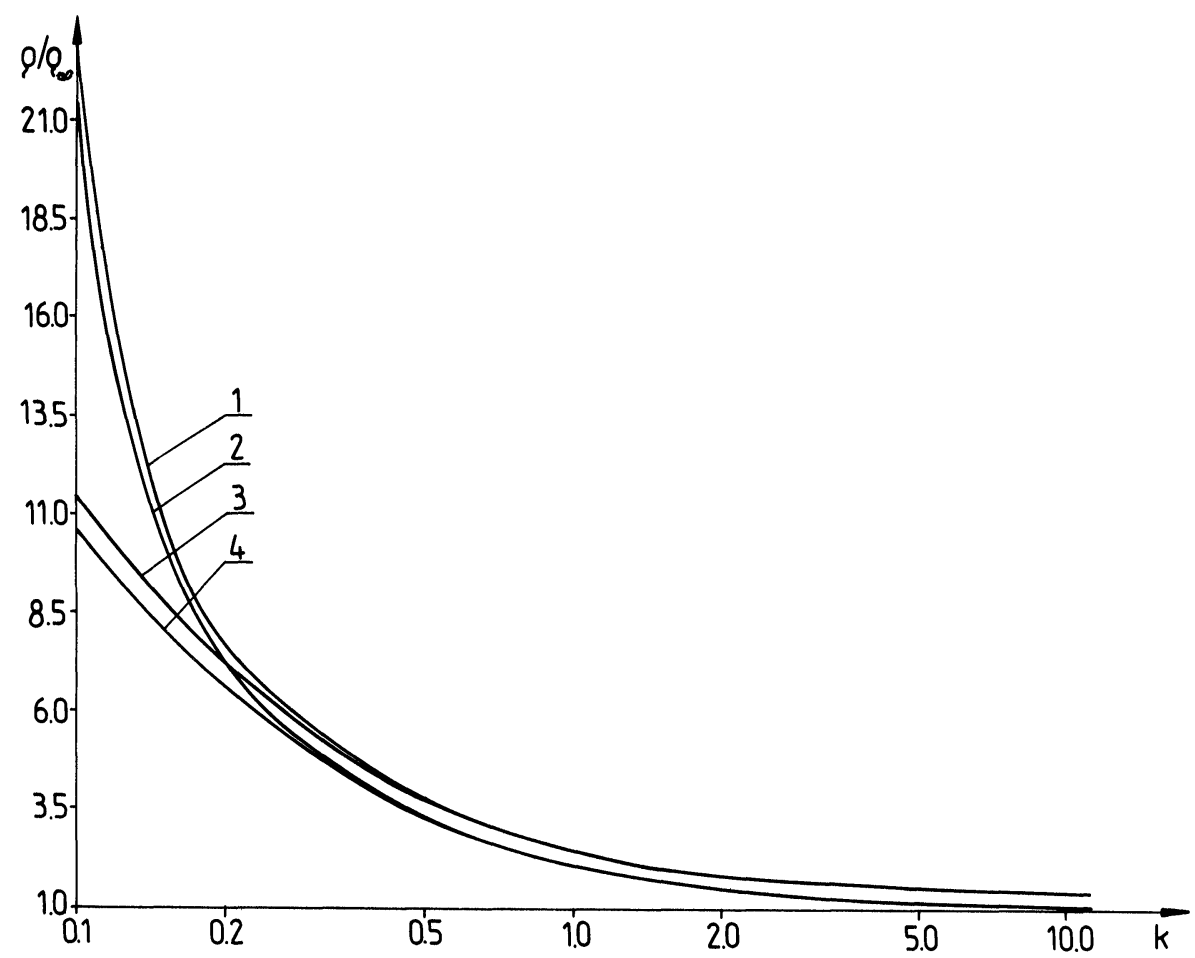

FIGURE 1 The electrical resistivity of thin metallic wires of a circular cross-section as a function of the diameter curve 1 for $R^{*}=0.2, T=1.0, B=0.05$ and $H=1.0$; curve 2 for $R^{*}=0.0, B=0.05$ and $H=1.0$; curve 3 for $\mathrm{R}^{*}=0.2 . \mathrm{T}=1.0 . \mathrm{B}=0.0$ and $H=1.0$; curve 4 for $\mathrm{R}^{*}=0.0 . \mathrm{B}=0.0$ and $\mathrm{H}=1.0$. 
at the beginning, electron scattering at the outer surfaces of the wire (so called: external size effect), taking account of the angular change of the specularity parameter, $\mathrm{p}(\theta)$, for electrons (Eq. (3)).

The parameter $\mathrm{p}(\theta)$ is dependent upon $\mathrm{h} / \lambda_{\mathrm{e}}$ where $\mathrm{h}$ is a root-mean-square surface roughness, and $\lambda_{\mathrm{e}}$ denotes the electron wavelength. In metals $\lambda_{\mathrm{e}}<\lambda_{\infty}$, where $\lambda_{\infty}$ is a mean free path of electrons in bulk metals and thus the parameter $\mathrm{p}(\theta)$ is not sensitive to the surface roughness. The mirror-like reflection takes place when the roughness of the outer surface is less than the electron wavelength. When considering the external size effect only an expression for electric conductivity, Eq. (5), has been obtained. Eq. (5) does not, however, describe entirely the electric conductivity of practical thin wires. Further, the expressions for conductivity or resistivity have been derived taking account of grain structure of the wire (Section 2.1.) and macroscopic roughness treated as a wire-diameter fluctuation (Section 2.2). The results obtained are presented in Eqs. (9) and (20). Eq. (9) can become Eq. (5) when we neglect the influence of electron scattering at grain boundaries. It is also easy to notice that if the wire-diameter fluctuations (i.e. $\mathrm{B}=\mathrm{g} / \lambda_{\infty}=0$ ) are neglected, then Eq. (20) becomes Eq. (9), i.e., it describes the resistivity of a thin wire taking account of external size effect and grain boundaries. A plot of $\rho / \rho_{\infty}$ versus $\kappa\left(=2 \mathrm{a} / \lambda_{\infty}\right)$ i.e. the ratio of wire diameter and mean free path of electrons is presented in Figure 1. The calculations have been performed assuming $\mathrm{H}=\mathrm{h} / \lambda_{\mathrm{e}}=1.0$ and the grain diameter $\mathrm{T}=\mathrm{D} / \lambda_{\infty}=1.0$. When comparing curves 1 and 3 with 2 and 4 , respectively, it can be seen how $\rho / \rho_{\infty}$ changes due to the grain boundaries for different wire thicknesses, $\mathrm{k}$. When $\mathrm{R}^{*}$ increases (electron reflection all grain boundaries), $\rho / \rho_{\infty}$ increases as well. It should be noticed that for $\mathrm{R}^{*}=0$ we have $\alpha=0$ and $\tau_{\mathrm{D}}=\tau$ according to Eq. (8).

When we compare curves 1 and 2 with 3 and 4 , respectively, we can see a significant change in the resistivity of thin wires as a function of the wire diameter fluctuations $\left(B=g / \lambda_{\infty}\right)$, especially for very thin wires (i.e. $\left.k<0.3\right)$. Hence, the wire diameter fluctuations, unavoidable in practice, ${ }^{7,10-12}$ are essential for examining very thin wires. We have assumed the sinusoidal fluctuations of the wire diameter (Eq. (11)). In the plots 1 and 2. (Figure 1) $\mathrm{B}=0.05$ has been assumed, and plots 3 and 4 are given for $\mathrm{B}=0$ (no fluctuation).

The calculations performed and discussion have led us to draw the following conclusions:

1) Grain boundaries greatly influence the electrical conductivity of wires. The more difficult an electron transition through the grain boundaries is, and the higher the grain density per unit volume is, then the higher is the wire resistivity.

2) The electrical conductivity of thin wires depends upon the wire-diameter fluctuation along the wire length. This is particularly true for thin wires.

Recently an interest in electron-transport investigations in fine wires both lithographically produced ${ }^{10-12}$ and drawn, ${ }^{7}$ has been renewed. These wires exhibit a significant defectiveness which influences their electrical resistivity. They are studied because a new type of ultrathin conductors exhibiting one-dimensional effects of electronical localization ${ }^{13}$ at very low temperatures are now possible. The three-dimensional transport theory presented has taken account of both electron scattering at barriers perpendicular to the electric current direction (here, called "grain boundaries", but these can also be flat defects) and the influence of the wire-diameter fluctuations on the wire conductivity, seems to be a satisfactory starting point for the investigation of the departure from three-dimensional Boltzmann transport of fine wires. 


\section{ACKNOWLEDGEMENTS}

This study was sponsored by the Institute of Physics of the Warsaw University, under Contract MR I-5. Thanks are expressed to Professor C. Wesolowska for her supervision.

\section{REFERENCES}

1. D.C. Larson, in: Physics of Thin Films, vol. 6, (Academic Press, New York, 1971).

2. R.B. Dingle, Proc. Roy. Soc. A201, 545 (1950).

3. J.R. Sambles, K.C. Elsom and T.W. Preist, J. Phys. F. 12, 1169 (1982).

4. J.M. Ziman, Electrons and Phonons, (Oxford University Press, London, 1960).

5. S.B. Soffer, J. Appl. Phys. 38, 1710 (1967).

6. A.F. Mayadas and Shatzkes, Phys. Rev. B. 1, 1382 (1970)

7. A.C. Sacharoff, R.M. Westervelt and J. Bevk, Phys. Rev. B. 26, 5976 (1982).

8. Y. Namba, Jap. J. Appl. Phys. 9, 1326 (1970).

9. K.C. Elsom and J.R. Sambles, J. Phys. F 11, 647 (1981).

10. J.T. Masden and N. Giordano, Phys. Rev. Lett. 49, 819 (1982).

11. G.J. Dolan and D.D. Osheroff, Phys. Rev. Lett. 43, 721 (1979).

12. P. Chaudhari and H.V. Habermeier, Phys. Rev. Lett. 44, 40 (1980).

13. D.J. Thouless, Phys. Rev. Lett. 39, 1167 (1977). 

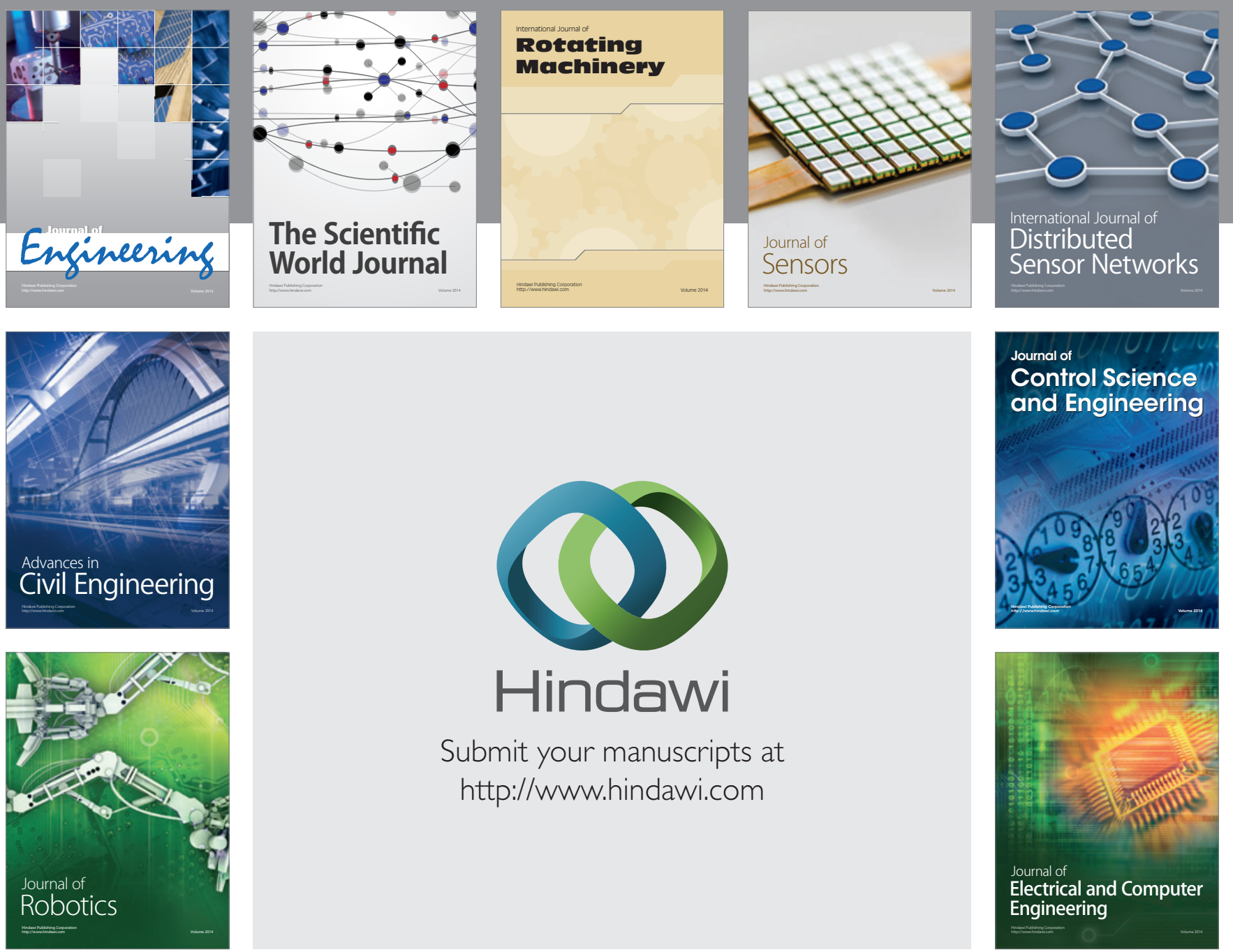

Submit your manuscripts at

http://www.hindawi.com
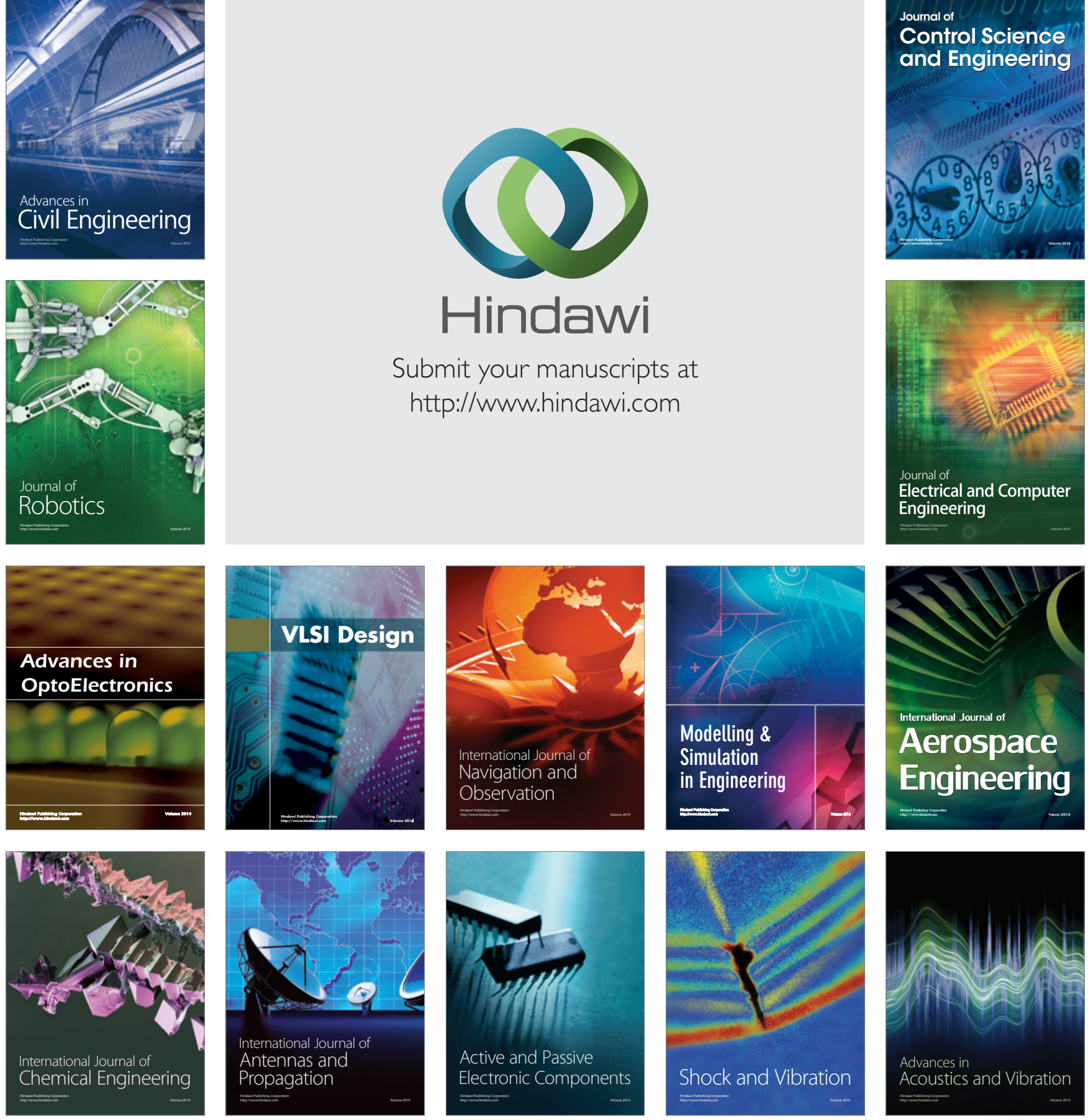